Otorhinolaryngol Nova 1994;4:112

\title{
Kongresskalender
}

\section{6.-18.6.1994 Utrecht}

The Netherlands

8.9.-11.9.1994 Arlington, Va.

USA

12th International Course in Functional Corrective Nasal Surgery

(preceding the ERS-ISIAN Congress in Copenhagen)

4th Research Workshop on the Biology, Prevention, and Treatment of Head and Neck Cancer Theme: International forum for the presentation and discussion of current research results from clinical trials and laboratory studies on head and neck cancer (Abstract deadline 01.03.1994) Auskunft: Prof. Dr. E.H. Huizing, Dept. of ORL, University Hospital Utrecht, PO Box 85500, NL-3508 GA Utrecht, Tel. 3230 506645, Fax 3230541922

Auskunft: Mrs Ruth Enquist, We Plan Meetings Inc., 1503 15th Street NE, Rochester, MN 55904323, USA, Fax 0015072818328

\section{6.-25.6.1994 München}

\section{9.-10.9.1994 Graz}

Österreich

12.9.-16.9.1994 Palma de Mallorca

Spanien

24.9.1994 Stollberg

Münchener Grundkurs für Allergologie

Nasenendoskopie, endoskopische Diagnostik und operative Technik für Anfänger und

Fortgeschrittene

Teilnahme an Sezierübungen möglich

1. Kongress der Deutsch-Spanischen HNO-Gesellschaft

Das hals-nasen-ohrenärztliche Gutachten Leitung: Dr. med. habil. Frey

Auskunft: Dr. A.G. Kühn, HNO-Klinik der Technischen Universität, Klinikum rechts der Isar, Ismaningerstr. 22, D-81675 München, Tel. 089/4140-2370, Fax 089/4180-5153

Auskunft: Arbeitsgemeinschaft Endoskopie, Prof. Dr. H. Stammberger, Univ. HNO-Klinik, Auenbruggerplatz 20, A-8036 Graz, Fax (++43) 3163853425

A. Auskunft: Hospital Universitario Son Dureta, Univ.-HNO-Klinik, Dr. M. Beraal-Sprekelsen, Andrea Doria, 55, E-07014 Palma de Mallorca, Tel. (0034) 71/175000 ext. 76100, Fax /175500 Auskunft: Sekretariat Dr. Frey, Kreiskrankenhaus Stollberg, Haus 2, Jahnsdorfer Str. 7, D-09166 Stollberg, Tel. 03729653216 